\title{
Hard- and Software Architecture of a Small Autonomous Underwater Vehicle for Environmental Monitoring Tasks
}

\author{
Christoph Osterloh, Marek Litza and Erik Maehle
}

\begin{abstract}
This paper presents the design of the hardware and software architecture, the selected sensors, sensor data processing and first experimental results for a small autonomous underwater vehicle (AUV). The robot was designed as an inexpensive autonomous platform for collecting environmental data in inshore and coastal waters, stand-alone or in swarms. The utilised thrusters allow the AUV a high range of speed, and its light sensor and echo sounder a detection of obstacles. A microcontroller is used as the main processing unit. The software is distributed over specialised tasks scheduled by a small Real Time Multitasking Kernel. Based on the integrated sensors an avoid and submergence behavior are presented.
\end{abstract}

\section{INTRODUCTION}

The quality of waterbodies is essential for the health of people living in its neighbourhood. In the EU Water Framework Directive (WFD 2000/60/EC) the European countries commit to achieve 'good quality' of all EU inshore and coastal waters by 2015 [1]. To control the quality, suitable monitoring methods are required. Here automatic systems can save costs compared to e.g. divers collecting data or human operators working on remotely operated vehicles (ROV) [2]. Beyond stationary sensor networks AUVs are used for such environmental monitoring tasks.

In the majority of cases only a single vehicle is used, thus it takes a long time to scan a large area. A large coordinated swarm of AUVs could solve this task in a more time efficient way. Furthermore, the exact localisation of a single AUV can be improved due to informations from its swarm neighbors [3]. The natural redundancy of a swarm allows higher fault-tolerance, even in case of a complete breakdown of

Christoph Osterloh, Marek Litza and Erik Maehle Institute of Computer Engineering, University of Luebeck, Ratzeburger Allee 160, 23538 Luebeck e-mail: \{osterloh, litza, maehle\}@iti.uni-luebeck.de 
one vehicle. Otherwise a swarm can collect a lot of redundant data and thereby improve its quality.

But even small of-the-shelf AUVs like [4] are relatively expensive and still unprepared for cooperation in large swarms or teams. For this purpose we present in this work a small inexpensive AUV MONSUN I (MONitoring System and Underwater Navigation Robot) specialized for coordinated environmental monitoring and suitable for beeing used in a team.

The expandable design allows it to carry different sensor sets and adjust to different tasks. In contrast to the Serafina project [5] we will equip our AUV also with a ballast diving system for static diving. Thus the robot will be able to lay on the ground and collect data with minimal power consumption. This and the a high bandwidth of possible speeds allows the vehicle to achieve different tasks in an energy efficient way.

Apart from recognizing obstacles by photosensors, a camera for identifying team members is integrated. Studies and simulations in [6] and [7] have shown that a basic swarm behavior like flocking are already possible without explicit communication only by the recognition of other robots and obstacles.

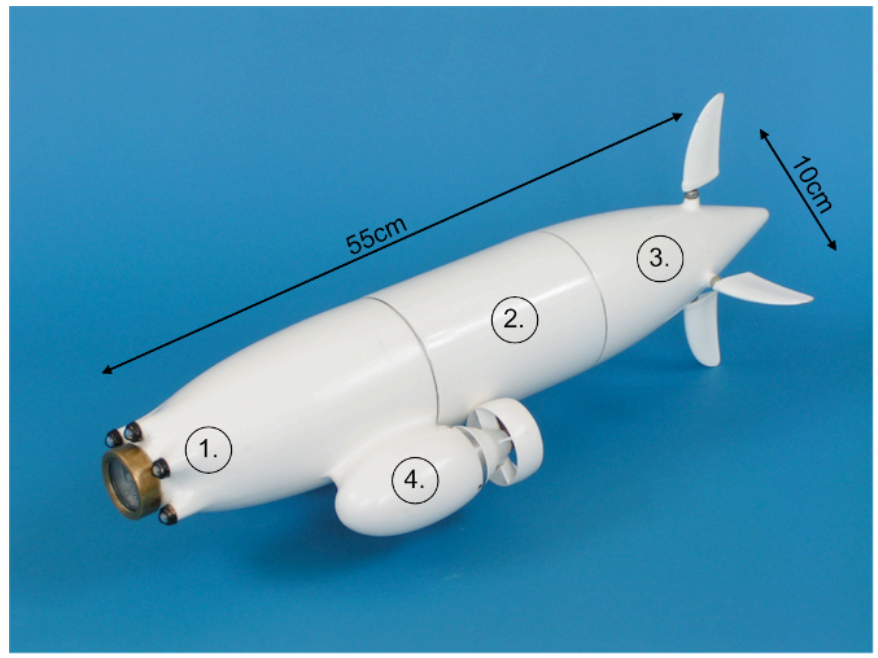

Fig. 1 The AUV MONSUN has a length of $55 \mathrm{~cm}$ and a width of $10 \mathrm{~cm}$ (without the thrusters). Part (1) contains the boards and the camera, part (2) the batteries and balast for balancing and the stern part (3) the servo motors for the rudders and elevators. The motors are housed in part (4).

The following section contains information about the hardware components and architecture of the AUV. Section 3 describes the robots software architecture and its behaviour. The experiments and its results are shown in Section 4 followed by the conclusions. 


\section{HARDWARE ARCHITECTURE}

\subsection{Mechanical and Electrical Systems}

The hull of the AUV has a length of $55 \mathrm{~cm}$ and a width of $10 \mathrm{~cm}$ (without motors). It is made of glass fibre reinforced plastic(GRP), and is divded in three parts (Fig. 1).

The front part has a viewing panel for the integrated camera. Five ultrabright white LEDs ( $25000 \mathrm{mcd}$ ) are arranged around the viewing panel, which are used for distance measurement by the light-sensor.

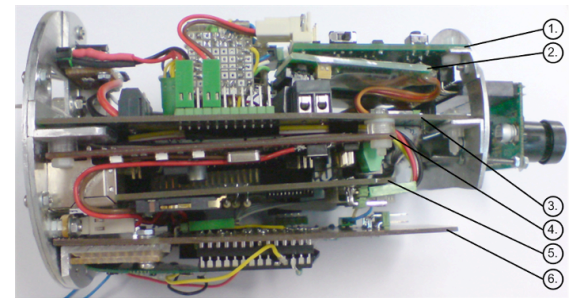

(a) Boards of MONSUN in the side view.

1. The CMUcam 3

2. Motor controller for the thrusters

3. Communication and navigation board

4. Ethernut board (main processing unit)

5. Logging, SD20 and navigation board

6. Fishfinder board

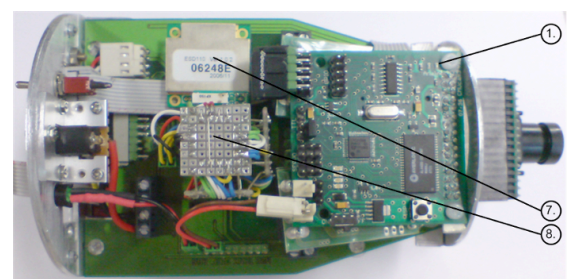

(b) Boards of MONSUN in the plan view.

1. The CMUcam 3

7. Bluetooth modul

8. Gyroscope cube

Fig. 2 Side and plan view of the boards of MONSUN.

Fig. 2 shows the boards of MONSUN, which are mounted on an aluminium frame, one above the other. Underneath the CMUCAM 3 camera module (Fig. 2(a) 1.), which is placed on top of the boards, the communication- and navigation board (Fig. 2(a) 3.) is located, including the motor controller for the thrusters (Fig. 2(a) 2.), the Bluetooth-module (Fig. 2(b) 7.) and the gyroscope cube (Fig. 2(b) 8.). Among the main processing unit (Fig. 2(a) 4.), a board for the servo-motor controller for the fins, the accelerometers and an SD card slot are arranged (Fig. 2(a) 5.). The bottommost board (Fig. 2(a) 6.) contains a second microcontroller for the echo sounder as well as power converter and a bus converter for the light sensor that is attached behind the viewing panel. Additionally there is a further SD card slot for the camera. Above the SD card slot, a small board for the echo sounder is mounted.

This arrangement was chosen to prevent long cable route and many connectors. The frame containing the boards can be assembled outside the AUV and fit in as one part. At the backside of the retainer a programming interface as well as a serial interface is located to reprogram the main processing unit while the boards are still incorporated within the hull. 
The power supply unit with accumulators and power converters is located in the $15 \mathrm{~cm}$ long middle part. Additionally there is place for two piston tanks for static diving, which momentarily is used to store balast for balancing.

In the stern part there are three servo-motors for the four movable fins. Furthermore, there are antagonistic weights to counteract the high weight of The AUVs front section. By connecting these three part by aluminium bayonet-mounts, the hull is pressure-resistant up to a depth of $10 \mathrm{~m}$.

The main thrusters of the AUV are reversible electric motors with $43 \mathrm{~mm}$ propellers, which generate $7 N$ of thrust and high range of speed. Therefore fast rotations with a small turning radius are possible, beside the commonly used forward or backward motion.

To steer the course and for dynamic diving we mounted four movable fins at the stern hull of the AUV. While the two rudders are connected by a linkage and are steered by one servo-motor, the two elevators are separately controllable. The servomotors have a torsional moment of $30 \mathrm{NCM}$ and a control speed of $0.16 \mathrm{~s} / 60^{\circ}$. Using the rudders it is possible to adjust small course changes and work against drift. The elevators allow dynamic diving as well as adjusting of possible inclinations in the water.

In order to avoid the generation of PWM signals for the motor and servo contol, we use a $S D 20$-servo-controller, which is connected to the control-platform by the Inter-Integrated Circuit Bus $\left(I^{2} C\right)$. This allows 126 discrete positions in both directions for each servo-motor, as well as 126 diskrete speed steps in both directions for the thrusters.

The electronic is supplied by two series-connected $7.4 \mathrm{~V}$ LiPo-batteries with $5.4 \mathrm{Ah}$. Supply voltages for the controller platform and sensors of $8 \mathrm{~V}, 5 \mathrm{~V}$ and $3 \mathrm{~V}$ are provided by potential transformers. The power supply is switched on in a contactless way with a solenoid in the rear part of the AUV.

As controller platform we use an Ethernut-Board 2.1B [8]. This board applies an 8 Bit ATmega128 running at $14.7456 \mathrm{MHz}$ with $128 \mathrm{~KB}$ flash memory and $4 \mathrm{~KB}$ SRAM and provides another $512 K B$ banked SRAM and $512 K B$ serial flash memory. Fig. 3 shows the connections of the different components and sensors to the ATmega128.

\subsection{Sensors}

The AUV MONSUN contains various sensors which can be divided into (1) positioning and navigation of the AUV and (2) assessment and storage of data.

For navigation unter water it is important to know the position, orientation, pitch and roll of the AUV. Moreover, to measure the distance to the ground and to detect obstacles are crucial features.

To track the position, orientation, pitch and roll of the AUV, we use a small Inertial Measurement Unit composed of a cube, on which three inexpensive ADIS16100 gyroscopes are located. These gyroscopes have a resolution of $0.24^{\circ} / \mathrm{s}$, a full-scale 


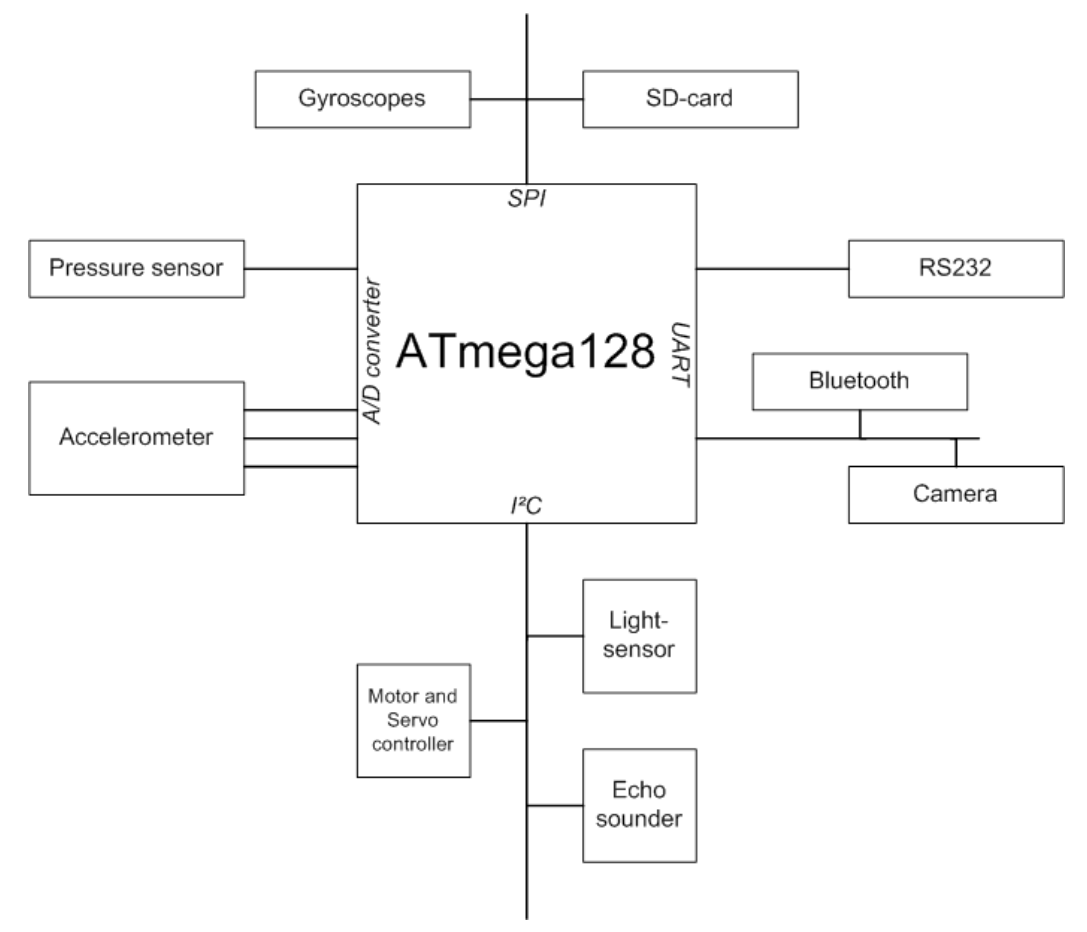

Fig. 3 Busnetwork connection hardware components to the ATmega128.

range of $\pm 300^{\circ} / \mathrm{s}$ and a bandwidth of $40 \mathrm{~Hz}$. Each gyroscope is equipped with a temperature sensor with a resolution of $0.14 K / L S B$. The gyroscopes are connected to the main processing unit by the serial peripheral interface (SPI). Additionally, we use a three-axis LIS3LO2AS4 accelerometer, which has a user selectable full-scale of $2 \mathrm{~g}$ or $6 \mathrm{~g}$. It is capable of measuring accelerations over a maximum bandwidth of $4.0 \mathrm{KHz}$ for the $X$ and $Y$ axis and $2.5 \mathrm{KHz}$ for the $Z$ axis. The sensor is adjusted to $2 \mathrm{~g}$ and the outgoing voltage is sampled and digitized with the internal $10 \mathrm{Bit}$ Analog/Digital converter of the ATmega128 with a frequency of $20 \mathrm{~Hz}$.

To measure depth, we use a Freescale MPXH6250A series pressure sensor with high temperatur accurancy. It has a range from 3.0 to $36 \mathrm{psi}$. The voltage output of the pressure sensor is connected to the A/D-converter of the ATmega128. The resolution of 10Bit enables an accuracy in depth measurement to $2.5 \mathrm{~cm}$.

The recognition of obstacles underwater is more difficult than in the air due to the characteristics of the water. In [10] some light-to-frequency sensors are employed. To solve this problem we use a TAOS TSL2561 light-to-digital converter that transform light intensity into a digital signal output equipped with an $I^{2} C$ interface. The integrated A/D-converter has a resolution of 16 Bit. The light-sensor ist combined with the five ultrabright white LEDs $(25000 \mathrm{mcd})$ in front of the AUV.

Because of the short range of the light-sensor of about $60 \mathrm{~cm}$ we integrated an echo sounder for distances above this value. Therefore we use a Fishfinder 
Humminbird SMARTCAST RF45E. This module has a sonar coverage of $90^{\circ}$, and is able to measure depth from $60 \mathrm{~cm}$ up to $30 \mathrm{~m}$. The measuring accuracy amounts about $4 \mathrm{~cm}$. Additionally it is possible to estimate the size of the objects by the length of the returning signal.

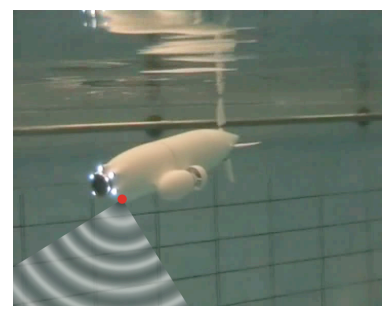

Fig. 4 Position and opening angle of the Echo Sounder in MONSUN

The piezo of the fishfinder is mounted in the front part of the AUV in an angle of $45^{\circ}$ to the longitudinal axis. This allows a detection of obstacles in front of or under the AUV (Fig. 4). Ordinarily, the fishfinder is a wireless sensor, which sends his data up to $30 \mathrm{~m}$ to the receiver. In order to process the data in the AUV, we removed the antenna and tap the sensor data before the transmission unit to compute the distance. This is done by a separate microcontroller (ATmega168), which acts as a slave and is connected to the main processor by the $I^{2} C$ Bus.

With the integrated CMUCAM 3 camera it is possible to take pictures from the AUV point of view. The filenames of the pictures contain the system time of the main processing unit, so pictures can be synchronized with the logging data. The camera is controlled by the main processing unit over a serial interface.

During a mission collected data concerning the position, behaviour and visualization need to be stored. For this purpose a SD card was integrated into the AUV. Data are stored in a text file, such that the records can be read out later. Additionally it is possible to send the records via Bluetooth to a receiver. Fig. 5 shows the struc-

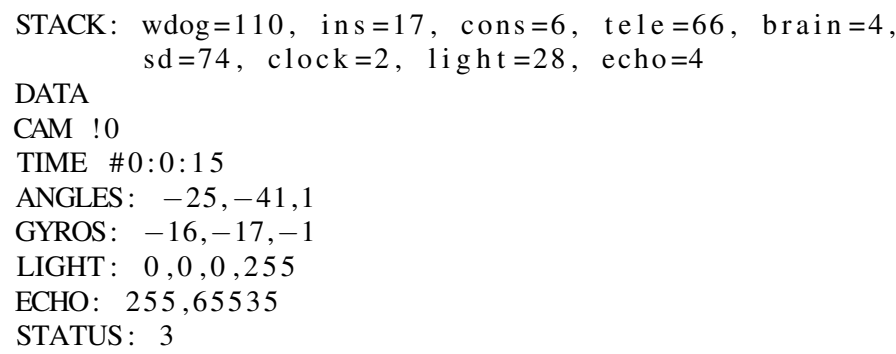

Fig. 5 Section of the logged data. The first two lines show the stack consumption of the running tasks. The next lines contains the instructions for the camera and the global system time. Among this, data of different sensors as well as the actual system state are stored. 
ture of a log file entry. Every five seconds the stack consumption of the individual tasks is written. Subsequently, the data of the acceleration sensors, the gyroscopes, the light-sensor, as well as the echo-sounder follow. Furthermore, actual instructions for the camera, the system time and the system status are dumped.

Data storage on a SD card is inflexible and does not allow to interfere with the AUV. At the water surface or close to it, it is possible to circumvent this aspect via a Bluetooth connection to a base station. MONSUN uses a Class 1 Bluetooth ESD110 module with a range of approx. $100 \mathrm{~m}$ and transmits data with up to $230 \mathrm{Kbps}$.

\section{SOFTWARE ARCHITECTURE}

The Software Architecture of MONSUN can be divided into six major units. The first part is the sensor unit, including the accelerometer and the gyroscopes, the lightsensor, echo sonar and pressure sensor for measuring depth and detecting obstacles. Although the sensors belong to one group, every sensor has his own task. The second unit are the actors. The servo and motor controller are part of this group. The third unit consists of the Inertial Navigation System (INS). The communication modules with the RS232 and Bluetooth interface represent the fourth part. The fifth unit is responsible for logging the sensory data for eventual analyses. The last unit is the high level control block of the AUV, including the main control unit and the behavior unit.

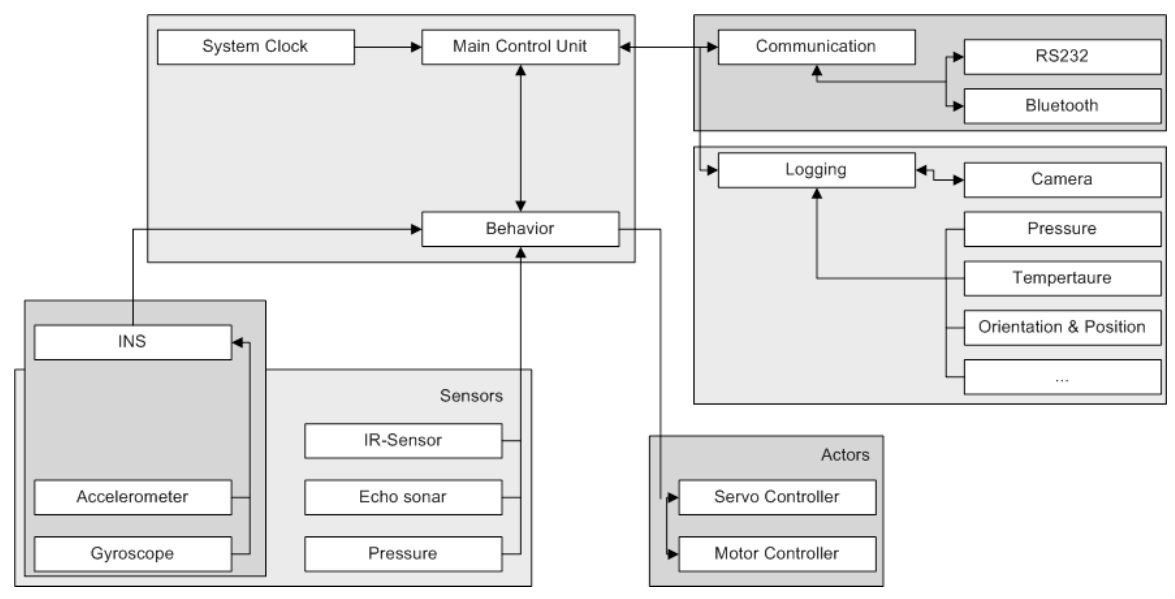

Fig. 6 The six parts of the Software Architecture of MONSUN.

Each of these tasks and sensors has different requirements in view of execution time and periodicity. Therefore, AvrX, a Real Time Multitasking Kernel written for the Atmel AVR series of micro controllers, is applied [9]. The Kernel is written in assembly language and has a size of approximately 700 words and is compatible 
with Gnu GCC and IAR compilers. AvrX contains approximately 40 functions for Tasking Management, Semaphores, Timer Management, Message Queues, Single Step Debugging support an FIFO support with synchronization.

\section{Behaviour}

MONSUN possesses different autonomous behaviors to avoid and submerge obstacles. Fig 7 shows a simple avoid behavior. When detecting an obstacle by its light sensor, MONSUN accelerates backwards and turns thereby around clockwise for about $90^{\circ}$ and then dives on.
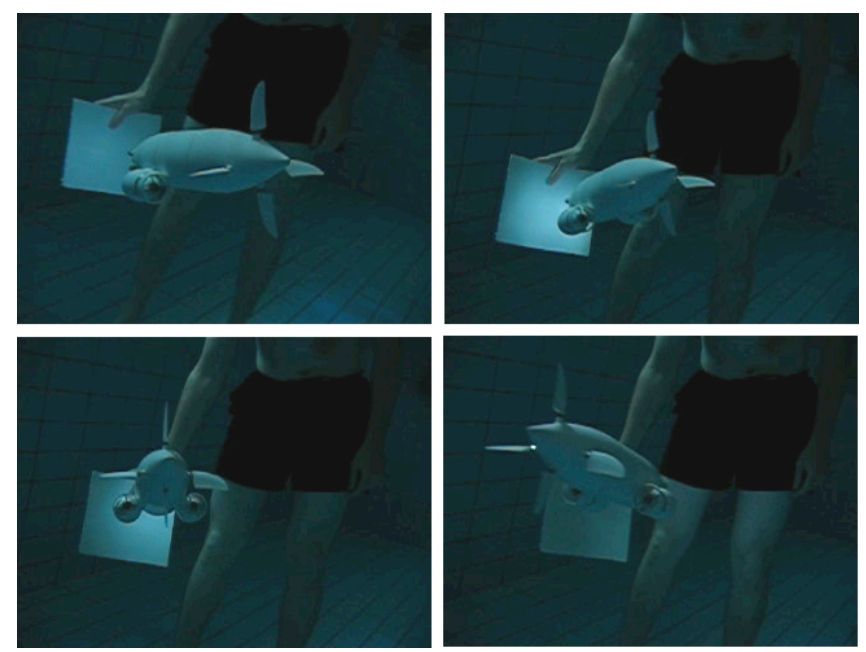

Fig. 7 The simple avoid behaviour of MONSUN. After detecting an obstacle, MONSUN accerelates backwards, turns around and dives on.

Fig. 9 shows a submerging of a recognised obstacle. Since currently MONSUN can submerge only dynamically, it accelerates backwards, also in order to prevent a collision with the obstacle while drifting. Afterwards, MONSUN dives by setting the fins and a strong acceleration forwards. If the lower end of the obstacle is recognised, the fins are set straight. After passing the object the emerging process begins. Its length depends on the time of submerging, in order to achieve the desired submerged depth again.

To verify the scenario shown in fig. 8 , we performed a test run. The sequence was filmed and tracked afterwards on the computer.

Fig. 9(a) shows the distance covered over time. As one can see, the obstacle was recorgnised at about second 3.25 and MONSUN accelerates backwards. At second 4.5 the thrusters change their direction and accelerate forwards to submerge the object. 


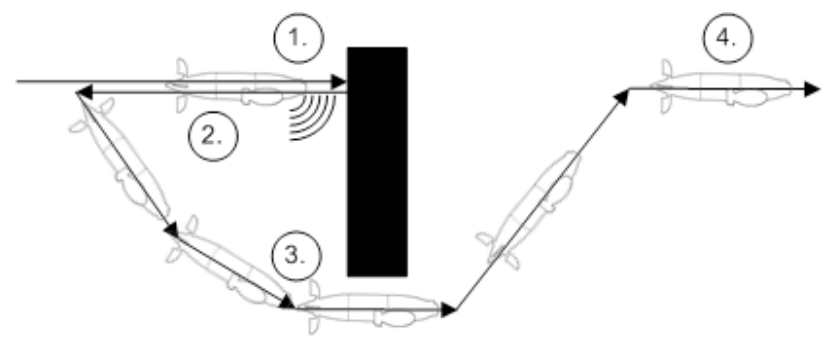

Fig. 8 Abstract representation of the programmed submergence scenario. MONSUN recognises the obstacle (1), steers backwards (2) and dives under (3) it to the original depth (4).

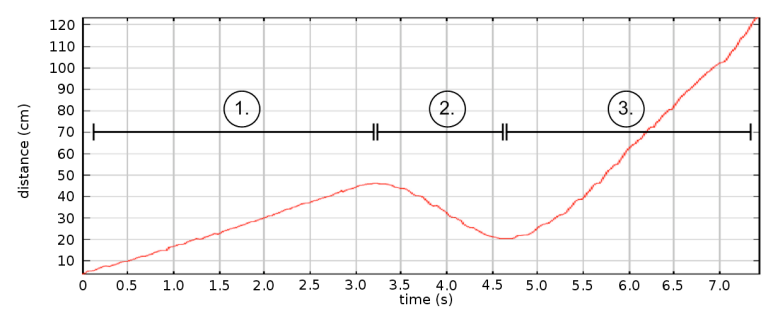

(a) Distance covered over time of a submergerce scenario. Phase (1) shows the forward motion and regognition of the obstacale, phase (2) the backward motion and phase (3) the submergence.

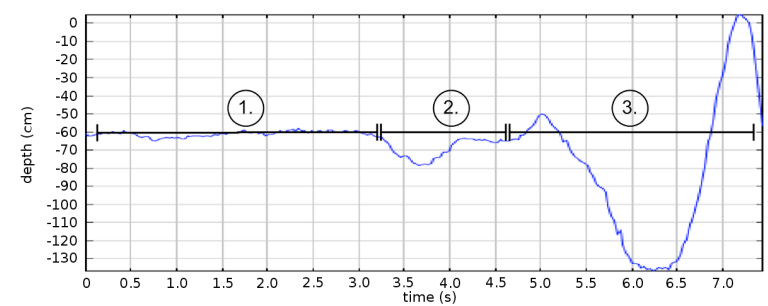

(b) Depth covered over time of a submergerce process. Phase (1) shows the forward motion and regognition of the obstacale, phase (2) the backward motion and phase (3) the submergence.

Fig. 9 Submergence scenario of MONSUN. Data were assessed by tracking the movement of MONSUN

Fig. 9(b) shows the depth covered over time. After detecting the obstacle, the fins are placed upwards. This causes the dip of the front part in the interval of time 3.25-3.75s. Because of the changing direction of the thrusters the front part of the AUV turns up briefly at time 5.0s before submerging the object. 


\section{CONCLUSIONS}

In this paper we presented the basic of a swarm competend AUV: MONSUN I. We have reported the hardware and software architecture and have shown, that MONSUN is capable to react to its environment by avoiding and submerging objects and obstacles. The high range of speed allows the fast reaching of a location as well as slow speed mobility for investigations. By recognising other team members it is (generally) possible to manage simple tasks without an explicit communication in the swarm.

The actual disadvantage of MONSUN I are mainly the choise of the some of its sensors and its hardware packaging. Some analog sensors, as for example the gyroscopes and acceleration sensors are too inaccurate for an exactly determination of the current position. The used hardware packaging makes it very difficult to replace individual components.

\section{FUTURE WORKS}

To avoid the drawbacks of MONSUN I, we currently redesign our AUV. The next generation MONSUN II incorporates an improved hardware and software architecture based on more accurate sensors and a modular packaging. More sensors for environmental measurement will be integrated as well as a piston tank for being able to collect data laying on the bottom of waters without using the thrusters.

For a better identification of the team members an improved camera unit is used. By additional light signals at the side and the rear end of MONSUN II, a better recognition of the team members and a relative positioning will be possible. In order to allow explicit communication between the team members, we are developing a small underwater modem. By this the robots will be able to exchange necessary environmental information or status messages among themselves coordinating the swarm more effectively.

\section{References}

1. The EU Water Framework Directive - integrated river basin management for Europe. http://eur-lex.europa.eu/LexUriServ/LexUriServ.do?uri=OJ:L:2000:327:0001:0072:EN:PDF

2. Listak, M.; Martin, G.; Pugal, D.; Aabloo, A.; Kruusmaa, M. (2005). Design of a semiautonomous biomimetic underwater vehicle for environmental monitoring. 6th IEEE International Symposium on Computational Intelligence in Robotics and Automation (CIRA 2005); Espoo, Finland; 27.06.-30.06.2005. New York: IEEE, 2005, 9 - 14.

3. Kottege, Navinda and Zimmer, Uwe R.: Relative localisation for AUV swarms in IEEE International Symposium on Underwater Technology 2007 (SUT '07), Tokyo, Japan,. pages 588-593, 2007

4. REMUS 100 description. http://www.hydroidinc.com/remus100.html 
5. The Serafina project. http://users.rsise.anu.edu.au/ serafina/

6. Arkin, R.C., 1992. Cooperation without Communication: Multi-agent Schema Based Robot Navigation, Journal of Robotic Systems, Vol. 9(3), pages 351-364

7. Geunho Lee and Nak Young Chong: Adaptive self-configurable robot swarms based on local interactions, Proceedings of the 2007 IEEE7RSJ Interantional Conference on Intelligent Robots and Systems, San Diego, CA, USA, Oct 29 - Nov 2, 2007, pages 4182-4187

8. Ethernut Board: Technical specification and datasheet. http://www.ethernut.de/pdf/ethernut21b_ehwm_2_0.pdf

9. AvrX Real Time Kernel specification. http://www.barello.net/avrx/

10. Jan Albiez, Jochen Kerdels, Sascha Fechner and Frank Kirchner: Sensor Processing and Behaviour Control of a Small AUV 20. Fachgespräch Autonome Mobile Systeme AMS 2007 Kaiserslautern, Kaiserslautern, Germany, Springer, pages 327-333, 2007 